\title{
BMJ Open Relative value of cystatin C and creatinine-based estimates of glomerular filtration rate in predicting long-term mortality after cardiac surgery: a cohort study
}

\author{
John F Mooney, ${ }^{1}$ Bernard L Croal, ${ }^{2}$ Sean Cassidy, ${ }^{2}$ Vincent W Lee, ${ }^{3}$ \\ Clara K Chow, ${ }^{1,4,5}$ Brian H Cuthbertson, ${ }^{6}$ Graham S Hillis ${ }^{\bullet 7,8}$
}

To cite: Mooney JF, Croal BL, Cassidy S, et al. Relative value of cystatin $C$ and creatinine-based estimates of glomerular filtration rate in predicting long-term mortality after cardiac surgery: a cohort study. BMJ Open 2019;9:e029379. doi:10.1136/ bmjopen-2019-029379

\section{- Prepublication history for} this paper is available online. To view these files please visit the journal online (http://dx.doi org/10.1136/bmjopen-2019029379).

Received 24 January 2019 Revised 25 July 2019 Accepted 08 August 2019
Check for updates

(C) Author(s) (or their employer(s)) 2019. Re-use permitted under CC BY-NC. No commercial re-use. See rights and permissions. Published by BMJ.

For numbered affiliations see end of article.

Correspondence to Professor Graham S Hillis; graham.hillis@health.wa.gov.au

\section{ABSTRACT}

Objective Renal dysfunction predicts an increased risk of both early and long-term mortality after cardiac surgery. Cystatin $\mathrm{C}$ enables glomerular filtration rate (GFR) to be estimated accurately and may be superior in this regard to creatinine-based estimates. We hypothesised, therefore, that cystatin $\mathrm{C}$ and derived estimates of GFR would independently predict long-term survival after cardiac surgery and would be superior in this respect to traditional estimates of GFR. The current study tests this hypothesis in a large and well-characterised cohort of patients. Design A prospective cohort study.

Setting Regional cardiothoracic centre in Northeast Scotland.

Participants 1010 patients undergoing non-emergent cardiac surgery between 2004 and 2007. Serum creatinine and cystatin $\mathrm{C}$ levels were measured preoperatively and demographic and clinical variables were recorded.

Primary outcome measure All-cause mortality, established from the National Records of Scotland. Results The median duration of follow-up after surgery was 9.7 years (IQR 8.9-10.6 years), during which 297 participants died. Preoperative creatinine and cystatin $\mathrm{C}$ levels and estimates of GFR derived from these were all strong predictors of death using Cox regression and remained independently predictive after adjustment for the logistic European System for Cardiac Operative Risk Evaluation, a well-validated clinical risk score and a range of other clinical predictors. Cystatin C-based measures were superior to creatinine-based estimates of GFR. Conclusions Cystatin $\mathrm{C}$ and creatinine derived eGFR are powerful and independent predictors of long-term mortality following cardiac surgery. Estimates of GFR derived from cystatin $\mathrm{C}$ convey superior prognostic information to conventional creatinine-based estimates, but the observed differences are modest.

Renal dysfunction prior to cardiac surgery is associated with an increased risk of perioperative complications and increased short-term and long-term mortality; ${ }^{1-4}$ and is an integral component of preoperative risk scores that are
Strengths and limitations of this study

- This study reports the outcome of a large and well-characterised cohort of patients who underwent cardiac surgery between 2004 and 2007 and is one of the longest follow-up studies assessing the prognostic importance of measures of renal function in this setting.

- The size of the cohort, duration of follow-up and high event rate (all-cause mortality) ensures that the study has considerable statistical power to address the relative ability of cystatin $\mathrm{C}$ to predict death following cardiac surgery.

- The study was, however, performed at a single regional cardiac surgical centre.

- Other limitations include the lack of a prospective screening log of patients who declined to participate in the study and the lack of data on important outcomes other than death.

commonly used in this setting. ${ }^{5-7}$ In routine clinical practice, measurement of renal function has traditionally relied on serum creatinine levels. These can then be used to derive the estimated glomerular filtration rate (eGFR), which is a more precise measure. ${ }^{8}$ The Chronic Kidney Disease Epidemiology Collaboration (CKD-EPI) equation has been adopted as a more accurate measure of eGFR, particularly mild renal dysfunction, and as a replacement to the Modification Diet in Renal Disease (MDRD) equation. ${ }^{9}$ Cystatin C is an alternate marker shown to be more accurate at detecting mild levels of renal dysfunction than creatinine. ${ }^{10}$ In contrast to serum creatinine, cystatin $\mathrm{C}$ is a protein released at constant rate from nucleated cells and is independent of muscle mass, age, gender and race and excreted exclusively by glomerular filtration. Blood levels of cystatin C are, 
therefore, an excellent indicator of renal function. ${ }^{10}$ They predict all-cause mortality and cardiovascular outcomes in a range of clinical settings including the general population, ${ }^{11}$ in populations with stable cardiovascular disease, ${ }^{12}$ after acute coronary syndrome,$^{13}$ in the elderly ${ }^{14}$ and in patients with heart failure. ${ }^{15}$ In addition, the use of cystatin $\mathrm{C}$ in combination with creatinine to estimate the GFR has been found to improve classification of chronic kidney disease in comparison to estimations of the GFR derived from either marker separately. ${ }^{16}$ Nevertheless, cystatin $\mathrm{C}$ is a relatively expensive test and is not routinely performed in clinical practice. Some studies have demonstrated that preoperative or early postoperative cystatin C levels are stronger predictors of acute kidney injury (AKI) following cardiac surgery than creatinine or creatinine-based estimates of GFR. ${ }^{17-19}$ Other large studies have, however, reported that cystatin $\mathrm{C}$ is less sensitive and detects AKI later than serum creatinine. ${ }^{20}$ The ability of preoperative cystatin $\mathrm{C}$ levels to predict medium to long-term survival in patients undergoing adult cardiac surgery has been less extensively investigated but appears promising. ${ }^{19} 21$ The current study tests the hypothesis that cystatin $\mathrm{C}$ and derived estimates of GFR are superior predictors of long-term all-cause mortality after cardiac surgery than creatinine-based estimates.

\section{METHODS}

\section{Trial design}

This was a retrospective analysis of a prospective single centre observational cohort study of 1010 consecutively recruited adult patients undergoing non-emergent cardiac surgery in Aberdeen, Scotland between January 2004 and August 2007.

\section{Participants}

The study population has been described previously. ${ }^{2223}$ All patients undergoing major cardiac surgery were eligible. There were no exclusion criteria. A screening log was not kept but all patients undergoing elective surgery were asked to participate and all those who provided consent are included in the study and these analyses.

\section{Measures and outcome}

Presurgical characteristics were collected from each patient. These included age, gender, weight, height, cardiovascular risk factors, medications and preoperative creatinine. Preoperative left ventricular systolic function (ejection fraction $<$ or $\geq 50 \%$ ) was recorded and the preoperative ECG was reviewed by a consultant cardiologist and designated as normal (no significant abnormality) or abnormal. The additive and logistic European System for Cardiac Operative Risk Evaluation (EuroSCORE) ${ }^{524}$ for each patient was also calculated. Intraoperative variables collected included the duration of cardiopulmonary bypass and aortic cross clamp times. Postoperative data collection included the duration of ventilation and the length of stay in intensive care and hospital. A small number of missing data points are listed in the footnote of table 1 . These were not imputed.

The primary endpoint of this study was all cause mortality. Vital status and date of death, if applicable, were determined from the National Records of Scotland.

The baseline eGFR was determined using the preoperative serum creatinine level, and calculated with the CKD-EPI equation..$^{25}$ As a single equation, it is expressed as $\mathrm{GFR}=141 \mathrm{x} \min (\mathrm{sCr} / \kappa, 1)^{\alpha} \mathrm{x} \max (\mathrm{sCr} / \kappa, 1)^{-1.209} \mathrm{x}$ $0.993^{\text {age }} \times 1.018$ (if female) $\times 1.159$ (if black), where $\mathrm{sCr}$ is serum creatinine, $\kappa$ is 0.7 for females or 0.9 for males, $\alpha$ is -0.329 for females or -0.411 for males, min indicates the minimum between $\mathrm{sCr} / \mathrm{\kappa}$ or 1 and max is the maximum value of $\mathrm{s} \mathrm{Cr} / \mathrm{\kappa}$ or 1 . For the comparison, we included the MDRD equation, ${ }^{26}$ which has been used in the literature in similar cardiothoracic populations, ${ }^{12}$ though in routine clinical practice, it has since been superseded by the CKD-EPI equation. Creatinine levels, measured between 2004 and 2007, were not standardised to isotope dilution mass spectrometry (IDMS). These were, therefore, recalibrated to IDMS standardised levels by reducing them by an adjustment factor of $5 \% .{ }^{97}$ These recalibrated values were used in all eGFR equations.

Preoperative serum samples were taken and serum cystatin C levels determined using an immuno-assay (Roche Diagnostics). Cystatin C was expressed in $\mathrm{mg} / \mathrm{L}$ and has an expected range of $0.47-1.09 \mathrm{mg} / \mathrm{L}$ in patients aged $20-70$ years. The eGFR derived from cystatin C levels was calculated using the method described by Grubb et $a l^{28}$ A separate equation that determines eGFR from both creatinine and cystatin $\mathrm{C}$ levels, and which has been found more accurate in general populations than eGFR derived from a single marker, was also used. ${ }^{16}$

\section{Statistical methods}

Categorical variables are presented numerically with percentages. Continuous variables are presented as mean values with $\mathrm{SD}$, if normally distributed, and as median and $\mathrm{IQR}$, if skewed. The normality of distribution of continuous variables was assessed using the Shapiro-Wilk test.

Estimates of risk were performed using Cox regression and expressed as HRs with 95\% CIs. Multivariable models were developed, also using Cox regression. In the first of these individual measures of renal function were entered along with the logistic EuroSCORE, a well-validated predictor of outcome after cardiac surgery that includes multiple variables, including creatinine values dichotomised as $>$ or $\leq 200 \mu \mathrm{mol} / \mathrm{L}$. A second model was developed that included independent predictors of mortality. For this analysis, an initial multivariable model was developed that included all potential clinical predictors of survival (univariable $\mathrm{p}<0.2)$, excluding measures of renal function. The independent clinical predictors $(\mathrm{p}<0.05$ in this multivariable model), thus, identified were then included in subsequent model that included individual measures of renal function. 
Table 1 Patient characteristics according to vital status at long-term follow-up

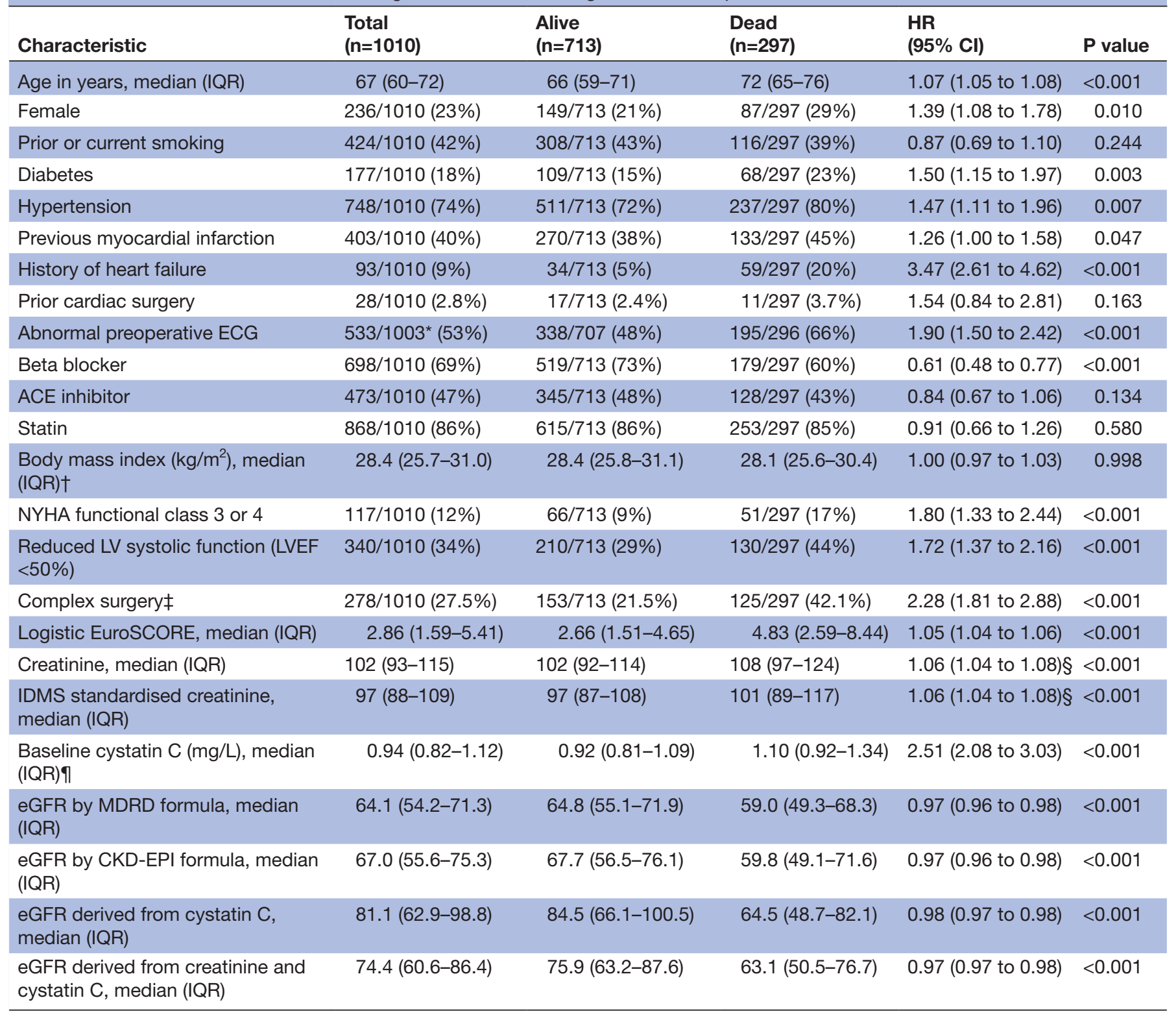

*One in seven individuals (six alive at follow-up and one deceased) the preoperative ECG was not available.

†In eight individuals (four alive at follow-up and four deceased) the preoperative body mass index was not recorded.

$\ddagger$ Valve and/or aortic surgery.

$\S \mathrm{HR}$ per $10 \mu \mathrm{mol} / \mathrm{L}$.

IIn nine cases (four alive at follow-up and five deceased) the preoperative cystatin C level was not available.

CKD-EPI, Chronic Kidney Disease Epidemiology Collaboration; eGFR, estimated glomerular filtration rate $\left(\mathrm{mL} / \mathrm{min} / 1.73 \mathrm{~m}{ }^{2}\right)$; EuroSCORE, European System for Cardiac Operative Risk Evaluation; IDMS, isotope dilution mass spectrometry; LVEF, left ventricular ejection fraction; MDRD, Modification Diet in Renal Disease; NYHA, New York Heart Association.

Levels of cystatin $\mathrm{C}$ were divided in to quarters based on the quartile values. In addition, patients were divided into stages of chronic kidney disease based on their GFR estimated using cystatin C. The risk of death in each group is presented using Kaplan-Meier survival curves and compared using the log rank test.

The ability of each measure of renal function to discriminate between those patients still alive at the end of follow and those who had died was assessed using receiver operating characteristic (ROC) curves, with calculation of the area under the curve and associated 95\% CIs. All participants contributed to these analyses. To simplify comparisons, the inverse value is presented for measures of eGFR (where the relationship to death is itself an inverse one, that is, a lower eGFR is associated with a higher risk). These curves were derived for each measure alone and, using binary logistic regression, for the combination of each measure with the logistic EuroSCORE. In addition, the areas under the dependent ROC curves were compared using the method of DeLong et al. ${ }^{29}$ 
Power calculations based on 30-day mortality have been previously published. ${ }^{22}$ We did not perform an a priori power calculation for this analysis. All analyses were conducted using SPSS software V.22 (IBM software) with the exception of the calculation and comparisons of the area under the ROC curves which were assessed using MedCalc V.19 (MedCalc; Ostend, Belgium). A p $<0.05$ was considered statistically significant.

\section{Patient involvement}

Patients were not involved in the design or conduct of this study.

\section{RESULTS}

The cohort included 1010 adult patients undergoing cardiac surgery, with preoperative serum cystatin C levels measured on 1001 patients. The overall distribution of patient characteristics according to vital status is shown in table 1.

The majority of surgical procedures were isolated coronary artery bypass grafting (CABG; 72\%), with the remainder undergoing valve surgery or a combination of both CABG and valve surgery. Using a cut-off of $<60 \mathrm{~mL} /$ $\min / 1.73 \mathrm{~m}^{2}$ calculated with the creatinine-based CKD-EPI equation, $42 \%$ of patients had chronic kidney disease. One patient was undergoing haemodialysis. Vital status was established in June 2016, representing a median follow-up of 9.7 years (IQR 8.9-10.6 years, range $0-11.8$ years). During this time, there were 297 deaths (29\%).

An increased hazard of death was observed in patients who were older, female and who had a prior history of hypertension, diabetes or heart failure (table 1). Higher long-term mortality was also observed in patients who required valve and/or aortic surgery, had an abnormal preoperative ECG, were in a higher New York Heart Association functional class (III or IV) or had impaired left ventricular systolic function. All measures of renal function (creatinine, cystatin $\mathrm{C}$ or eGFR calculated using the MDRD, CKD-EPI, cystatin C or combined creatinine and cystatin C-based equations) were also strong predictors of long-term survival.

The risk of death was particularly evident in patients who had cystatin C levels above the median level $(0.935 \mathrm{mg} / \mathrm{L})$, with the highest mortality of all observed among patients with levels in the highest quarter $(>1.120 \mathrm{mg} / \mathrm{L}$; figure 1 , $\log$ rank test $\left.\chi^{2} 77.5, \mathrm{p}<0.001\right)$. A similar pattern was observed when patients were divided on the basis of their stage of chronic kidney disease as determined using the GFR estimated using cystatin C (figure 2; log rank test $\chi^{2}$ 104.1, $\mathrm{p}<0.001)$.

The association between preoperative cystatin $\mathrm{C}$ or eGFR, however measured, and long-term survival remained significant after adjustment for the logistic EuroSCORE (table 2). Likewise, a significant association was evident after separate adjustment for selected clinical predictors of survival (age, history of diabetes, history of hypertension, prior myocardial infarction, history of cardiac failure, left ventricular ejection fraction $<50 \%$ and valve and/or aortic surgery; table 2).

The ability of each measure of renal function to predict long-term mortality after cardiac surgery was further assessed using ROC curves, both individually and in combination with the logistic EuroSCORE. Based on these curves, all measures of renal function exhibited a modest to good ability to discriminate between patients who were alive and those who had died during follow-up (table 3) but serum cystatin c and estimates of GFR derived from it were better discriminators (ie, had greater areas under the ROC curves) than the creatinine derived estimations of GFR. Likewise, for each measure of renal function, the area under the ROC curve was greater when it was combined with the logistic EuroSCORE (table 3). The GFR estimated using the CKD-EPI formula was a better discriminator of vital status than that derived using the MDRD formula (area under curve 0.641 vs 0.625 , $\mathrm{p}<0.001$ ) and the eGFR derived using cystatin $\mathrm{C}$ was superior to cystatin $\mathrm{C}$ levels alone (area under curve $0682 \mathrm{vs}$ $0.667, \mathrm{p}<0.001)$. Further comparisons between the creatinine-based CKD-EPI eGFR, the cystatin C-based eGFR and the eGFR derived from both creatinine and cystatin C confirmed that both the cystatin C-based eGFR and the eGFR derived from both creatinine and cystatin $\mathrm{C}$ were similar discriminators of survival and both were superior to the eGFR based on the CKD-EPI formula using creatinine alone (table 4).

The logistic EuroSCORE was a superior discriminator of mortality than GFR estimated using the CKD-EPI formula but not using either estimate that included cystatin C (table 4). However, the combination of the cystatin C-derived eGFR and logistic EuroSCORE (area under curve 0.721 ) conveyed similar prognostic information to the combination of eGFR derived from the CKD-EPI formula and the logistic EuroSCORE (area under curve 0.709, $\mathrm{p}=0.23$ for comparison of areas under the ROC curves).

The combination of the logistic EuroSCORE and GFR estimated using cystatin $\mathrm{C}$ has the highest area under the ROC curve of any combination (0.721) and was statistically superior to either parameter alone $(p=0.03$ for comparison with logistic EuroSCORE alone and $\mathrm{p}=0.006$ for comparison with cystatin C-derived eGFR alone). Preoperative levels of cystatin $\mathrm{C}$ alone were comparable discriminators of long-term mortality to the logistic EuroSCORE (table 3, $\mathrm{p}=0.118$ for comparison of areas under the ROC curves).

\section{DISCUSSION}

This study reports the relationship between preoperative renal function, measured in several ways, and subsequent mortality in a large and well-characterised cohort of patients who underwent cardiac surgery a median of 9.7 years previously. It is one of the longest available follow-up studies assessing the prognostic importance of renal function in patients undergoing cardiac surgery and confirms that this is a powerful discriminator of 


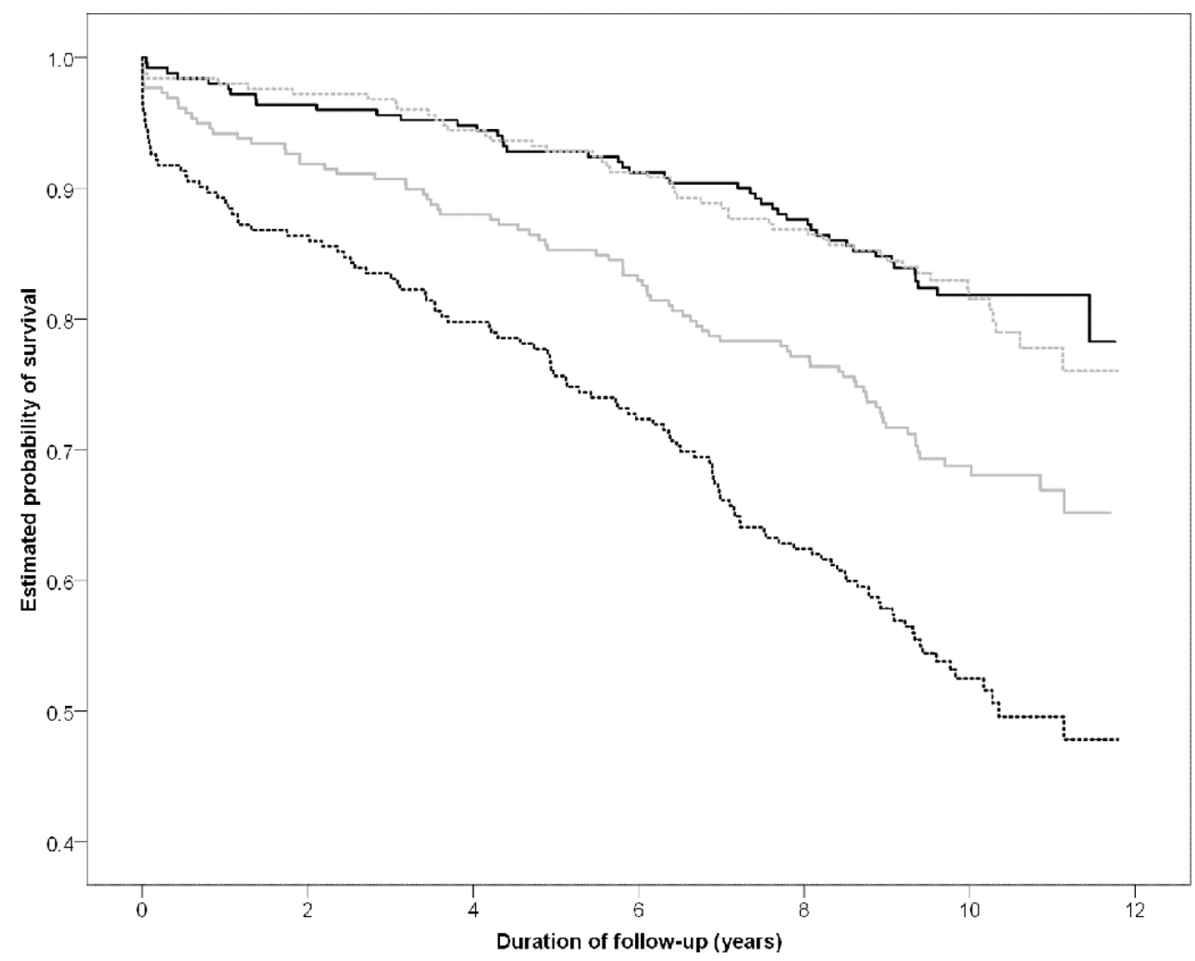

$\begin{array}{lllllll}\text { At risk } & \text { Yr 0 } & \text { Yr 2 } & \text { Yr 4 } & \text { Yr 6 } & \text { Yr 8 } & \text { Yr 10 } \\ \text { Quarter 1 } & 250 & 241 & 237 & 228 & 219 & 122 \\ \text { Quarter 2 } & 251 & 244 & 237 & 229 & 218 & 112 \\ \text { Quarter 3 } & 258 & 237 & 227 & 214 & 199 & 98 \\ \text { Quarter 4 } & 242 & 209 & 193 & 175 & 151 & 67\end{array}$

Cystatin C levels quarter 1 (lowest; < $0.815 \mathrm{mg} / \mathrm{L}$ )

Cystatin C levels quarter $2(0.815-0.935 \mathrm{mg} / \mathrm{L})$

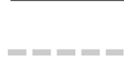

Cystatin C levels quarter $3(0.936-1.120 \mathrm{mg} / \mathrm{L})$

Cystatin C levels quarter 4 (highest; $>1.120 \mathrm{mg} / \mathrm{L}$ )

Figure 1 Survival after cardiac surgery according to levels of cystatin C.

long-term survival in this setting. Renal function, however measured, is strongly predictive of death and is independent of other well-recognised clinical predictors and a well-validated, widely used, risk score; despite this already including a crude measure of renal function (creatinine $>$ or $\leq 200 \mu \mathrm{mol} / \mathrm{L}$ ). In particular, the results suggest that levels of cystatin $\mathrm{C}$ and estimates of GFR derived from this are stronger and more accurate predictors of subsequent death than purely creatinine-based estimates of the GFR measured either by the MDRD formula or the more contemporary CKD-EPI formula. The current data also confirm that a single preoperative blood level of cystatin $\mathrm{C}$ is as good a discriminator of long-term survival after cardiac surgery as the 17 variable logistic EuroSCORE; though this does not imply that it should be used as a substitute for this proven clinical scoring system. Indeed, combining cystatin $\mathrm{C}$ levels or the derived eGFR with the logistic EuroSCORE modestly, but significantly, improves the prognostic information provided by either method (biochemical measurement or clinical risk score) alone.

Renal function is a particularly important predictor of outcome following cardiac surgery. ${ }^{40}$ In this setting the eGFR, derived from measurement of serum creatinine, is a powerful predictor of short and medium-term survival. Despite its theoretical advantage, there are limited data regarding the prognostic value of cystatin $\mathrm{C}$ in patients undergoing cardiac surgery; and those available have limitations and are inconclusive. Several groups have tested the hypothesis that cystatin $\mathrm{C}$ would be superior to creatinine in predicting AKI early after cardiac surgery with mixed results. ${ }^{20} 31$ Far fewer studies have explored the ability of preoperative cystatin C, and GFR estimated from this, to predict mortality in this setting and none have reported this over long-term ( $>5$ years) follow-up.

Ledoux et al studied 376 patients undergoing cardiac surgery in a single centre in Belgium and found that 


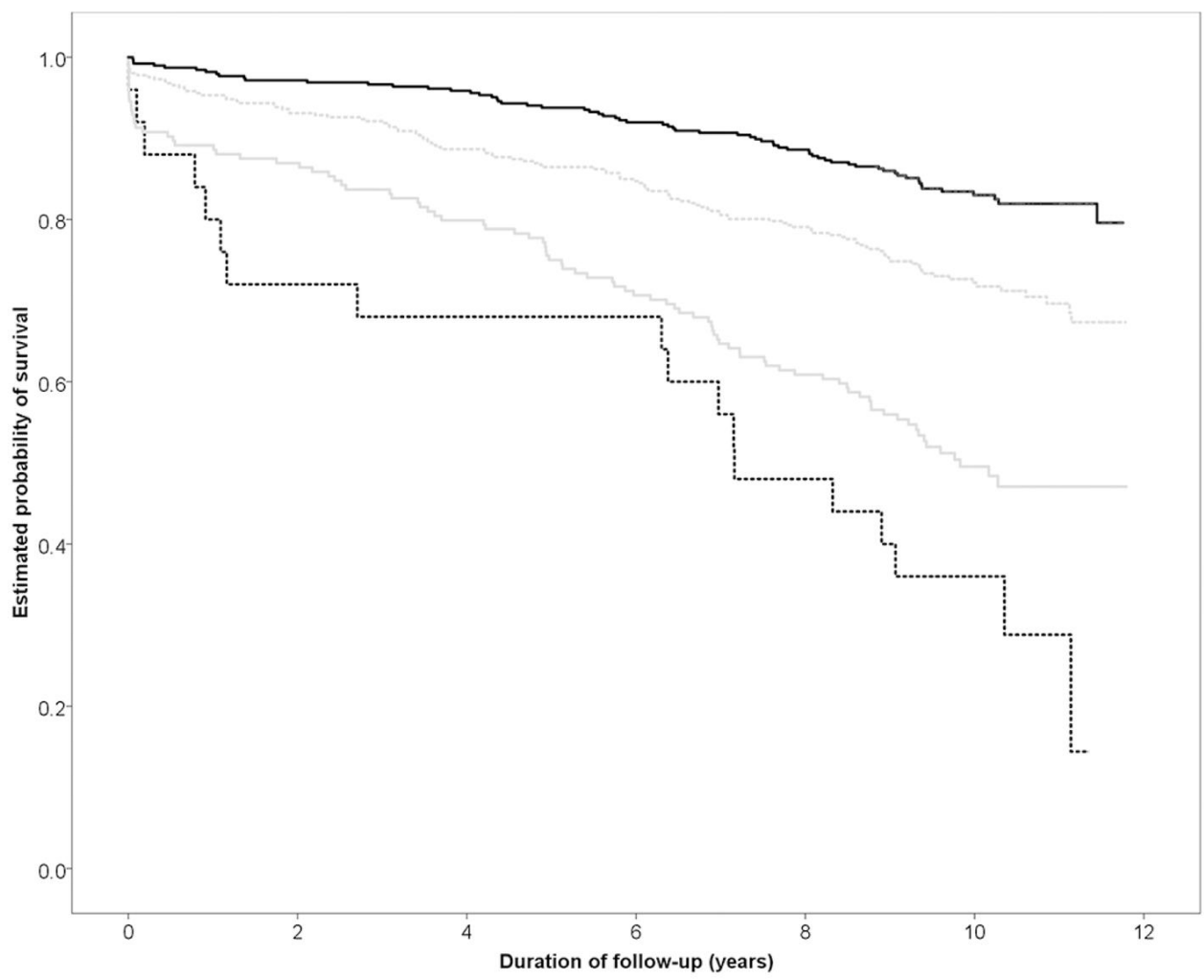

$\begin{array}{lcccccc}\text { At risk } & \text { Yr 0 } & \text { Yr 2 } & \text { Yr 4 } & \text { Yr 6 } & \text { Yr 8 } & \text { Yr 10 } \\ \text { CKD stage 1 } & 386 & 375 & 370 & 355 & 342 & 185 \\ \text { CKD stage 2 } & 406 & 378 & 360 & 344 & 321 & 157 \\ \text { CKD stage 3 } & 184 & 160 & 147 & 130 & 112 & 52 \\ \text { CKD stage 485 } & 25 & 18 & 17 & 17 & 12 & 5\end{array}$

CKD stage 1 based on glomerular filtration rate estimated using cystatin $C\left(\geq 90 \mathrm{~mL} / \mathrm{min} / 1.73 \mathrm{~m}^{2}\right)$

CKD stage 2 based on glomerular filtration rate estimated using cystatin C $\left(60-89 \mathrm{~mL} / \mathrm{min} / 1.73 \mathrm{~m}^{2}\right)$

CKD stage 3 based on glomerular filtration rate estimated using cystatin C (30-59 mL/min/1.73m²)

CKD stages 4 \& 5 based on glomerular filtration rate estimated using cystatin $C\left(<30 \mathrm{~mL} / \mathrm{min} / 1.73 \mathrm{~m}^{2}\right)$

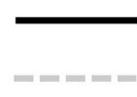

10

Figure 2 Survival after cardiac surgery according to levels of glomerular filtration estimated using cystatin C.

preoperative GFR estimated using cystatin $\mathrm{C}$ was associated with 1 year mortality $(\mathrm{n}=38,10 \%)$ and was superior in this respect than GFR estimated using the MDRD equation. ${ }^{21}$ Both measures were, however, strong predictors and the superiority of a predictive model that included cystatin $\mathrm{C}$ eGFR and the additive EuroSCORE over a model containing the MDRD eGFR and additive EuroSCORE was modest.$^{21}$ In 1033 patients who underwent off pump CABG and were followed up for a mean of just under 3 years cystatin $\mathrm{C}$ levels were superior to creatinine in predicting postoperative renal dysfunction, as was the cystatin C-derived GFR compared with the MDRD estimated GFR. Cystatin C predicted mortality $(n=55)$ with an area under the ROC curve of $0.73(95 \% \text { CI } 0.70 \text { to } 0.76)^{19}$; higher than we observed. In that study, in univariable analyses, both creatinine and cystatin C estimated GFR levels were predictors of mortality but neither remained significant predictors in multivariable analyses, perhaps reflecting the limited power of this study. ${ }^{19}$ The one other study that has assessed the relative ability of cystatin $\mathrm{C}$ and creatinine-derived estimates of GFR to predict the medium-term mortality after cardiac surgery studied 1638 patients from Sweden, followed up for a median of $31 / 2$ (range 2-5) years. ${ }^{32}$ This study reports that the eGFR derived from cystatin $\mathrm{C}$ had an area under the ROC curve of 0.805 (95\% CI 0.763 to 0.846 ) for the prediction of death $(n=114)$, much higher than GFR estimated using the CKD-EPI creatinine-based formula $(0.702,95 \%$ CI 0.649 
Table 2 The association between measures of renal function and long-term all-cause mortality

\begin{tabular}{lcccc}
\hline & Wald $\chi^{2}$ & HR & 95\% Cl & P value \\
\hline Adjusted for logistic EuroSCORE & & & & \\
\hline Serum cystatin C & 62.759 & 2.203 & 1.812 to 2.679 & $<0.001$ \\
\hline eGFR calculated using MDRD equation & 27.186 & 0.978 & 0.970 to 0.986 & $<0.001$ \\
\hline eGFR calculated using CKD-EPI equation & 37.378 & 0.977 & 0.969 to 0.984 & $<0.001$ \\
\hline eGFR calculated using cystatin C & 69.942 & 0.979 & 0.975 to 0.984 & $<0.001$ \\
\hline $\begin{array}{l}\text { Combined creatinine and cystatin C-derived eGFR } \\
\text { Adjusted for independent clinical predictors of survival }\end{array}$ & 63.759 & 0.975 & 0.969 to 0.981 & $<0.001$ \\
\hline Serum cystatin C & 15.848 & 1.707 & 1.312 to 2.221 & $<0.001$ \\
\hline eGFR calculated using MDRD equation & 4.388 & 0.991 & 0.982 to 0.999 & 0.036 \\
\hline eGFR calculated using CKD-EPI equation & 5.572 & 0.990 & 0.981 to 0.998 & 0.018 \\
\hline eGFR calculated using cystatin C & 13.246 & 0.989 & 0.984 to 0.995 & $<0.001$ \\
\hline Combined creatinine and cystatin C-derived eGFR & 11.543 & 0.988 & 0.981 to 0.995 & 0.001 \\
\hline
\end{tabular}

${ }^{*}$ Age, history of diabetes, history of hypertension, prior myocardial infarction, history of cardiac failure, left ventricular ejection fraction $<50 \%$ and valve and/or aortic surgery.

CKD-EPI, Chronic Kidney Disease Epidemiology Collaboration; eGFR, estimated glomerular filtration rate; EuroSCORE, European System for Cardiac Operative Risk Evaluation; MDRD, Modification Diet in Renal Disease.

to 0.755 ) or observed in the current study. ${ }^{32}$ The reasons for these discrepant results, in terms of the predictive accuracy, are not clear. There are differences in the study populations as Dardashti $e t$ al studied only patients undergoing CABG whereas the current study included patients undergoing valve and aortic surgery. That, however, does not seem to explain the findings as the area under the ROC curve for cystatin C-derived eGFR in our cohort was 0.637 in patients undergoing isolated CABG and 0.698 in patients undergoing more complex surgery. Another explanation might be the longer duration of follow-up in the current study. Again, however, we found no evidence to support this, with an area under the ROC curve for cystatin C-derived eGFR of 0.687 at 3 years.

\section{Strengths and weaknesses of the study}

This study has several strengths. Participants were prospectively recruited and well characterised. They have been followed up for many years using national death records, ensuring that vital status data are accurate and

Table 3 Ability of measures of renal function to predict long-term mortality using the respective areas under their receiver operating curves

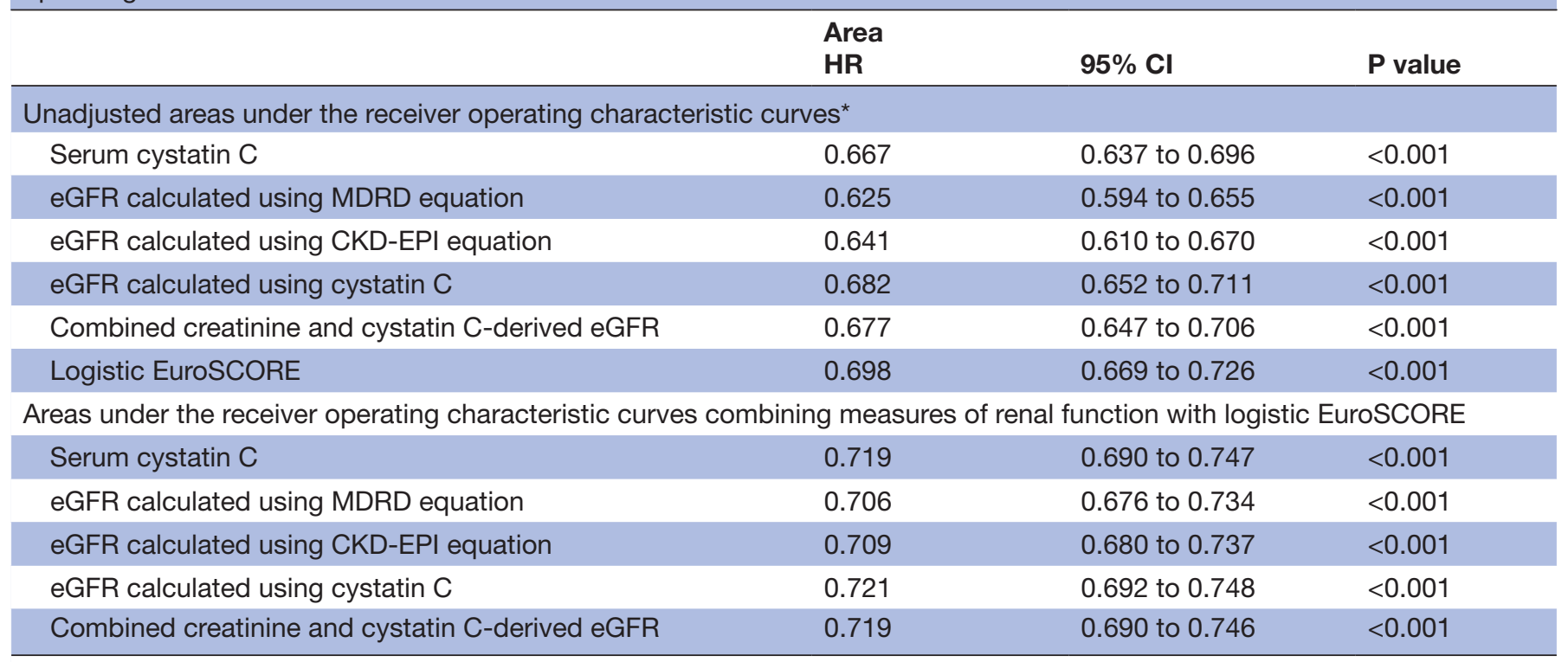

*To simplify comparisons the inverse value for the area above the receiver operating curve is presented for measures of eGFR.

CKD-EPI, Chronic Kidney Disease Epidemiology Collaboration; eGFR, estimated glomerular filtration rate; EuroSCORE, European System for Cardiac Operative Risk Evaluation; MDRD, Modification Diet in Renal Disease. 
Table 4 Selected comparisons of the areas under the receiver operating characteristic curves

\begin{tabular}{|c|c|c|c|}
\hline & Difference in AUC* & $95 \% \mathrm{Cl}$ & P value \\
\hline $\begin{array}{l}\text { eGFR calculated using cystatin C versus eGFR calculated using CKD-EPI } \\
\text { equation }\end{array}$ & 0.0390 & 0.0077 to 0.0703 & 0.0145 \\
\hline $\begin{array}{l}\text { eGFR calculated using cystatin C versus eGFR calculated using } \\
\text { combined creatinine and cystatin C }\end{array}$ & 0.0047 & -0.0088 to 0.0181 & 0.4954 \\
\hline EuroSCORE versus eGFR calculated using cystatin C & 0.0180 & -0.0205 to 0.0565 & 0.3589 \\
\hline $\begin{array}{l}\text { EuroSCORE versus eGFR calculated using combined creatinine and } \\
\text { cystatin C }\end{array}$ & 0.0226 & -0.0156 to 0.0608 & 0.2469 \\
\hline
\end{tabular}

*Slight differences from those calculable from table 3 are due to nine patients with missing cystatin $\mathrm{C}$ levels.

AUC, area under curve; CKD-EPI, Chronic Kidney Disease Epidemiology Collaboration; eGFR, estimated glomerular filtration rate; EuroSCORE, European System for Cardiac Operative Risk Evaluation.

complete. The size of the cohort and length of follow-up determine that many patients have died in the interim and ensures that the study has considerable statistical power. Indeed, the current study has greater power to address the relative ability of cystatin $\mathrm{C}$ to predict death following cardiac surgery than all prior studies that have explored this combined. The study also has limitations. A screening $\log$ of patients who refused to participate was not maintained, though anecdotally this was very rare and it seems unlikely this will have affected the study findings or conclusions. The study was confined to a single centre with the limits on generalisability that this confers. In addition, the study used only single preoperative measures of renal function (creatinine and cystatin C), which precludes any assessment of the stability of the levels. The study consisted, however, of stable patients and the main focus was to compare the different measures. The study also focused on a single outcome, all-cause mortality, and did not assess the relationship between renal function and specific causes of death (eg, cardiovascular) or other important outcomes such as the need for postoperative renal replacement therapy or major cardiovascular events.

\section{CONCLUSION}

This study confirms that preoperative renal function, regardless of how this is measured, is a strong predictor of long-term mortality after cardiac surgery, even after accounting for other clinical predictors. The results confirm the prognostic utility of cystatin $\mathrm{C}$ levels and the GFR estimated from them. However, the differences in terms of the prognostic information obtained were the modest when compared with creatinine-based measures, which are widely available, routinely performed and much cheaper. Thus, it is unlikely that routine measurement of cystatin $\mathrm{C}$ would be used for this purpose in everyday clinical practice. Whether the measurement of cystatin $\mathrm{C}$ is of value in predicting other important outcomes, such as postoperative AKI, remains unclear and is the subject of ongoing research. Likewise, further work is required to determine whether the inclusion of more precise, creatinine derived, estimates of renal function can improve clinical risk scores. Finally, the results suggest that optimising renal function in patients undergoing cardiac surgery and adopting an aggressive approach to the management of risk factors for worsening dysfunction might potentially improve long-term outcomes. This is another area worthy of further research.

\section{Author affiliations}

${ }^{1}$ Cardiovascular Division, The George Institute for Global Health, Sydney, New South Wales, Australia

${ }^{2}$ Department of Biochemistry, Aberdeen Royal Infirmary, Aberdeen, UK

${ }^{3}$ Westmead Institute for Medical Research, University of Sydney, Sydney, New South Wales, Australia

${ }^{4}$ Sydney Medical School, The University of Sydney, Sydney, New South Wales, Australia

${ }^{5}$ Westmead Applied Research Centre, Westmead Hospital, Sydney, New South Wales, Australia

${ }^{6}$ Critical Care Medicine, Sunnnybrook Health Sciences Centre, Toronto, Ontario, Canada

${ }^{7}$ Department of Cardiology, Royal Perth Hospital, Perth, Western Australia, Australia ${ }^{8}$ Faculty of Health and Medical Sciences, University of Western Australia, Perth, Western Australia, Australia

Acknowledgements We are grateful to the staff of the Cardiothoracic Surgical and Intensive Care Units of Aberdeen Royal Infirmary. In addition, we would like to thank Sally Burrows, Biostatistician, University of Western Australia, for her advice.

Contributors Conception and design of this study: $\mathrm{BeC}, \mathrm{BrC}$ and $\mathrm{GH}$. Acquisition of data: $\mathrm{BeC}, \mathrm{BrC}$ and $\mathrm{GH}$. Drafting of manuscript: JM and GH. Interpretation of data, critical revision of manuscript and final approval: JM, BeC, SC, VWL, CC, BrC and GH. Overall accountability for the work: GH

Funding This work was supported by The British Heart Foundation and Chest Heart and Stroke, Scotland.

Competing interests None declared.

Patient consent for publication Not required.

Ethics approval Ethics approval for the study was granted by the local research ethics committee prior to commencement.

Provenance and peer review Not commissioned; externally peer reviewed. Data availability statement Data are available on reasonable request.

Open access This is an open access article distributed in accordance with the Creative Commons Attribution Non Commercial (CC BY-NC 4.0) license, which 
permits others to distribute, remix, adapt, build upon this work non-commercially, and license their derivative works on different terms, provided the original work is properly cited, appropriate credit is given, any changes made indicated, and the use is non-commercial. See: http://creativecommons.org/licenses/by-nc/4.0/.

\section{REFERENCES}

1. Hillis GS, Croal BL, Buchan KG, et al. Renal function and outcome from coronary artery bypass grafting: impact on mortality after a 2.3year follow-up. Circulation 2006;113:1056-62.

2. Cooper WA, O'Brien SM, Thourani VH, et al. Impact of renal dysfunction on outcomes of coronary artery bypass surgery: results from the Society of thoracic surgeons national adult cardiac database. Circulation 2006;113:1063-70.

3. Holzmann MJ, Hammar N, Ahnve S, et al. Renal insufficiency and long-term mortality and incidence of myocardial infarction in patients undergoing coronary artery bypass grafting. Eur Heart $J$ 2007;28:865-71.

4. Mooney JF, Ranasinghe I, Chow CK, et al. Preoperative estimates of glomerular filtration rate as predictors of outcome after surgery: a systematic review and meta-analysis. Anesthesiology 2013;118:809-24.

5. Nashef SAM RF, Michel P, Gauducheau E, et al. The EuroSCORE Study Group. European system for cardiac operative risk evaluation (EuroSCORE). Eur J Cardiothorac Surg 1999;16:9-13.

6. Parsonnet V, Dean D, Bernstein AD. A method of uniform stratification of risk for evaluating the results of surgery in acquired adult heart disease. Circulation 1989;79:I3-12.

7. Shahian DM, O'Brien SM, Filardo G, et al. The Society of Thoracic Surgeons 2008 cardiac surgery risk models: part 1--coronary artery bypass grafting surgery. Ann Thorac Surg 2009;88(1 Suppl):S2-22.

8. Levey AS, Coresh J, Balk E, et al. National kidney Foundation practice guidelines for chronic kidney disease: evaluation, classification, and stratification. Ann Intern Med 2003;139:137-47.

9. Matsushita K, Mahmoodi BK, Woodward M, et al. Comparison of risk prediction using the CKD-EPI equation and the MDRD study equation for estimated glomerular filtration rate. JAMA 2012;307:1941-51.

10. Taglieri N, Koenig W, Kaski JC. Cystatin C and cardiovascular risk. Clin Chem 2009;55:1932-43.

11. Wu C-K, Lin J-W, Caffrey JL, et al. Cystatin C and long-term mortality among subjects with normal creatinine-based estimated glomerular filtration rates: NHANES III (third National health and nutrition examination survey). J Am Coll Cardiol 2010;56:1930-6.

12. Ix JH, Shlipak MG, Chertow GM, et al. Association of cystatin C with mortality, cardiovascular events, and incident heart failure among persons with coronary heart disease: data from the heart and soul study. Circulation 2007;115:173-9.

13. Jernberg $T$, Lindahl B, James $S$, et al. Cystatin C: a novel predictor of outcome in suspected or confirmed non-ST-elevation acute coronary syndrome. Circulation 2004:110:2342-8.

14. Shlipak MG, Sarnak MJ, Katz R, et al. Cystatin C and the risk of death and cardiovascular events among elderly persons. $N$ Engl J Med 2005;352:2049-60.
15. Sarnak MJ, Katz R, Stehman-Breen CO, et al. Cystatin C concentration as a risk factor for heart failure in older adults. Ann Intern Med 2005;142:497-505.

16. Inker LA, Schmid $\mathrm{CH}$, Tighiouart $\mathrm{H}$, et al. Estimating glomerular filtration rate from serum creatinine and cystatin C. $N$ Engl J Med 2012;367:20-9.

17. Shlipak MG, Coca SG, Wang Z, et al. Presurgical serum cystatin C and risk of acute kidney injury after cardiac surgery. Am J Kidney Dis 2011;58:366-73.

18. Koyner JL, Garg AX, Shlipak MG, et al. Urinary cystatin C and acute kidney injury after cardiac surgery. Am J Kidney Dis 2013;61:730-8.

19. Lee $\mathrm{SH}$, Youn $\mathrm{Y}-\mathrm{N}$, Choo HC, et al. Cystatin $\mathrm{C}$ as a predictive marker of renal dysfunction and mid-term outcomes following off-pump coronary artery bypass grafting. Heart 2015;101:1562-8.

20. Spahillari A, Parikh CR, Sint K, et al. Serum cystatin C- versus creatinine-based definitions of acute kidney injury following cardiac surgery: a prospective cohort study. Am J Kidney Dis 2012;60:922-9.

21. Ledoux D, Monchi M, Chapelle J-P, et al. Cystatin C blood level as a risk factor for death after heart surgery. Eur Heart J 2007;28:1848-53.

22. Cuthbertson BH, Croal BL, Rae D, et al. N-Terminal pro-B-type natriuretic peptide levels and early outcome after cardiac surgery: a prospective cohort study. Br J Anaesth 2009;103:647-53.

23. Cuthbertson BH, Croal BL, Rae D, et al. N-Terminal pro-Btype natriuretic peptide concentrations and long-term outcome after cardiac surgery: a prospective cohort study. $\mathrm{Br} J$ Anaesth 2013;110:214-21.

24. Michel P, Roques F, Nashef SAM, et al. Logistic or additive EuroSCORE for high-risk patients? Eur J Cardiothorac Surg 2003;23:684-7.

25. Levey AS, Stevens LA, Schmid CH, et al. A new equation to estimate glomerular filtration rate. Ann Intern Med 2009;150:604-12.

26. Levey AS, Bosch JP, Lewis JB, et al. A more accurate method to estimate glomerular filtration rate from serum creatinine: a new prediction equation. modification of diet in renal disease Study Group. Ann Intern Med 1999;130:461-70.

27. Levey AS, Coresh J, Greene T, et al. Expressing the modification of diet in renal disease study equation for estimating glomerular filtration rate with standardized serum creatinine values. Clin Chem 2007;53:766-72.

28. Grubb A, Nyman U, Björk J, et al. Simple cystatin C-based prediction equations for glomerular filtration rate compared with the modification of diet in renal disease prediction equation for adults and the Schwartz and the Counahan-Barratt prediction equations for children. Clin Chem 2005;51:1420-31.

29. DeLong ER, DeLong DM, Clarke-Pearson DL. Comparing the areas under two or more correlated receiver operating characteristic curves: a nonparametric approach. Biometrics 1988;44:837-45.

30. Hillis GS, Cuthbertson BH, Croal BL. Renal function, revascularization and risk. Eur Heart J 2007;28:782-4.

31. Zhang Z, Lu B, Sheng X, et al. Cystatin C in prediction of acute kidney injury: a systemic review and meta-analysis. Am J Kidney Dis 2011;58:356-65.

32. Dardashti A, Nozohoor S, Algotsson L, et al. The predictive value of $s$-cystatin $C$ for mortality after coronary artery bypass surgery. $J$ Thorac Cardiovasc Surg 2016;152:139-46. 